\title{
Study on the Shear Band of Sand with Various Particle Characteristics Using PIV Analysis
}

\author{
Shintaro Kajiyama ${ }^{1, *}$, Yukio Nakata ${ }^{1}$, Ryota Miyamoto $^{1}$ and Masato Taue ${ }^{1}$ \\ ${ }^{1}$ Yamaguchi University, Graduate school of science and technology for innovation, 2-16-1 Tokiwadai Ube Yamaguchi, Japan
}

\begin{abstract}
The phenomenon of ground undergoing large plastic deformation, leading to collapse, occurs due to the localization of unstable deformation. The investigation of shear band is important in order to understand the destructive phenomenon. Therefore, a series of experiments were conducted on sands with different particle characteristics so that behaviour of the shear band of sand under plane strain compression could be investigated. Specifically, Toyoura sand and two kinds of coral sand, hereafter referred to as coral sand A (with smaller average particle size) and coral sand B (with larger average particle size) were used. The shear band was evaluated using PIV (Partial Image Velocimetry) analysis. As a result, it became clear that the relationship between shear inclination angle and internal friction angle does not hold for the two kinds of coral sand because the shear inclination angle is low. The ratio of the average value of the shear band width to the average grain size at the peak was 10 to 20 times, 7 to 10 times, and 5 to 8 times in the order of Toyoura sand, coral sand A, and coral sand B. The sample standard deviation was 0.1 to $0.9 \mathrm{~mm}, 0.9$ to $1.6 \mathrm{~mm}$, and 1.4 to $1.9 \mathrm{~mm}$
\end{abstract}

\section{Introduction}

The phenomenon that the ground undergoes large plastic deformation and destroys occurs due to localization of unstable deformation. The area where localization concentrates is called the shear band. Detailed investigation of this shear band is dynamically important in capturing destruction phenomena. On the other hand, the behaviour of the ground is varied depending on the type of sand even at the same confining pressure and density. In particular, when crushable materials are used, which are prone to fracture individual particles, it is thought that the influence is remarkably in the shear band where shear stress is applied [1]. In grasping the shear band of crushable sand, since the principal stress direction is defined by one plane, it is necessary to measure the strength deformation characteristics under the plane strain condition using a plane strain compression apparatus. Therefore, in this study, a series of experimental studies were conducted with the aim of grasping the shear band of sand under plane strain compression, and using the PIV (Partial Image Velocimetry) analysis with picture of membrane which covered the specimen, the angle of the shear band and the width of the shear band study was carried out.

\section{Materials}

Two kinds of coral sand and Toyoura sand were used for this study. Figure 1 shows the photo of the sample used in this study taken by electronic microscope. Figure 2 shows the particle size distribution of these samples. As it can be seen from the figure, the two types of coral sands have different particle sizes, and for the average particle diameter $D_{50}$, the smaller one is $D_{50}=0.48 \mathrm{~mm}$, and the bigger one is $D 5_{50}=1.10 \mathrm{~mm}$. In addition, Toyoura sand has $D_{50}=0.22 \mathrm{~mm}$. Hereinafter, those having a small average particle size are referred to as coral sand A, and the larger one is referred to as coral sand B.

\section{Testing method}

\subsection{Test procedure}

In this research, a plane strain testing apparatus which can easily observe the shear band was used. The apparatus has an observation window that allows observation of the deformation situation during the test, so that the side of the specimen in the direction of the intermediate principal strain can be observed. Since the observation window is a flat surface, there is no distortion in the obtained image and it is only necessary to consider the influence of distortion of the lens of the camera at the time of analysis. A specimen was prepared as the relative density $\mathrm{Dr}=80 \%$ by using the air drop method. The size of the specimen is $160 \mathrm{~mm}$ in height, $60 \mathrm{~mm}$ in width and $80 \mathrm{~mm}$ in depth.

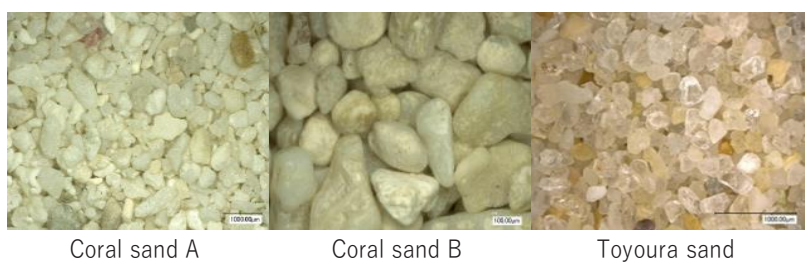

Fig. 1. Materials used in present study

\footnotetext{
*orresponding author: kajiyama@yamaguchi-u.ac.jp
} 


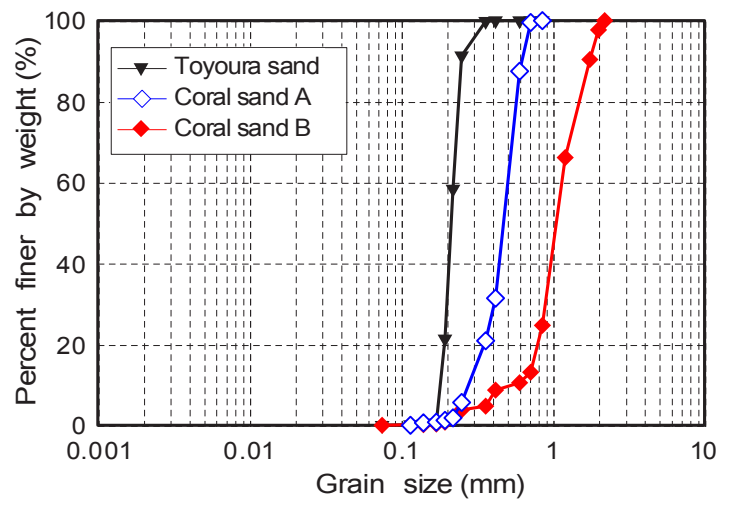

Fig. 2. Particle size distribution curve

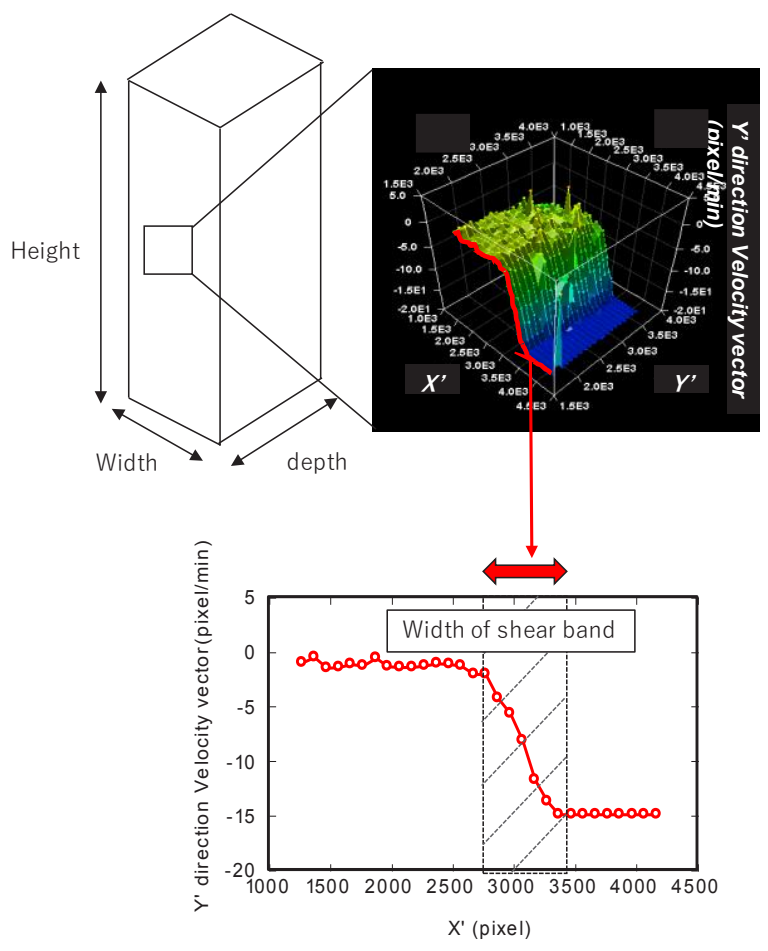

Fig. 3. Definition of acceleration distribution (upper) and width of shear zone obtained from PIV analysis (lower)

After installing the specimen, a back pressure B.P. $=30$ $\mathrm{kPa}$ was applied and the specimen was isotropically consolidated under a predetermined consolidation stress before static shearing was performed until the axial strain reached $10 \%$. The strain rate was $0.1 \% / \mathrm{min}$. The confining pressure is adjusted by air pressure, and cell pressure, back pressure, displacement is controlled by computer. Images of the entire specimen were acquired at $0.1 \%$ intervals and PIV analysis was performed. In addition, in order to investigate the degree of crushing of particles, a single particle crushing test [2] was performed on coral sand B. First of all, one particle of coral sand was placed on the loading platform and installed in the test equipment. Thereafter, the loading plate was lowered from the upper part, a load was applied to the single particle, and the load and the displacement amount were measured.

\subsection{Analysis procedure}

In this study, PIV analysis was used to obtain the displacement amount from the image. The shear band was defined based on PIV analysis result. For the extraction of the shear band, analysis was performed using an image obtained by photographing a part of the specimen so that the shear band comes to the center of the image, separately from the image obtained by shooting the entire specimen. Figure 3 shows the definition of shear band extraction. First, the coordinates of the upper and lower parts of the shear band are read from the geometrically corrected image to calculate the angle of the shear band. Next, the image is rotated so that the angle of the shear band becomes 90 degrees. PIV analysis was performed on a range wider than the shear band, with the horizontal direction of the rotated image taken as $X^{\prime}$ and the vertical direction as $Y^{\prime}$. The image used for the analysis is a shear band photographed every $0.1 \%$ axial strain at shearing. Since the shear band width is assumed to be 10 to 20 times the average particle diameter from the existing research, the size of one element is $100 \times 100$ (pixel) $(0.6 \mathrm{~mm} \times 0.6$ $\mathrm{mm})$. The photograph was coordinated by analysis and the difference between the coordinate at $\mathrm{t}+1$ (minute) and the coordinate at $\mathrm{Y}$ 'at $\mathrm{t}$ (minute) was found to obtain the velocity vector in $\mathrm{Y}^{\prime}$ direction. A graph of a velocity vector in the $\mathrm{Y}$ 'direction for each column was made and a region in which the slope of the minute section continued to be positive with the maximum value of the minute section of this curve as the center was defined as a shear band. The width of the shear band was calculated for each row and the average value of all rows was taken as the shear band width.

\section{Results and discussion}

First, the result of the single particle crushing test conducted to investigate the strength of single coral sand particles is shown. The single particle crushing test was performed on 100 particles of $0.25 \mathrm{~mm}$ to $0.75 \mathrm{~mm}$ which are close to the average particle diameter of coral sand B as a representative. Figure 4 shows the survival probability curve for single particle crush strength. The figure also shows the results of Toyoura sand [3]. It is clear from the figure that the characteristic strength (crushing strength at 37\%) of coral sand B is $19.9 \mathrm{MPa}$. Figure 5 shows the relationship between single particle crush strength and particle diameter before crushing. From the figure it is clear that the quartz sand of similar grain size is $60 \mathrm{MPa}$, whereas the strength is roughly $1 / 3$. Based on the above particle characteristics, a shear test was conducted.

Figure 6 shows the stress-strain relationship obtained by the experiment conducted with the effective stress $\sigma_{\mathrm{c}}{ }^{\circ}=$ $100 \mathrm{kN} / \mathrm{m}^{2}$. It is clear that the Toyoura sand has a stronger 


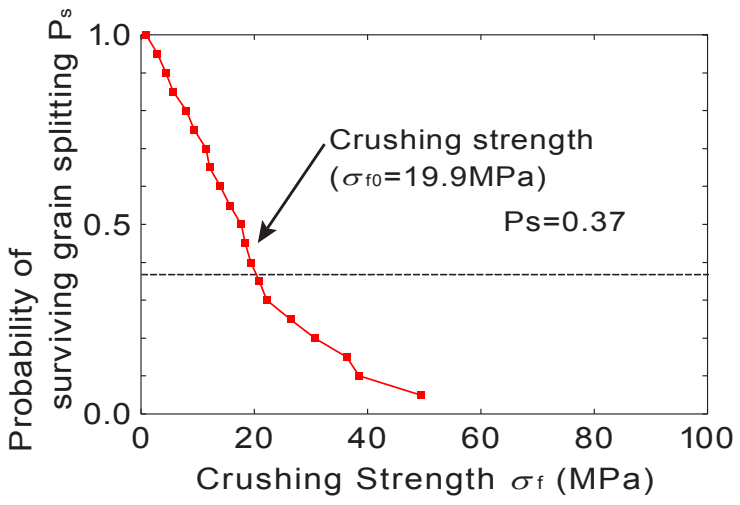

Fig. 4. Survival probability curve for single particle crushing strength

tendency to show a positive dilatancy than either sample of coral sand A or B which is a crushable material. Comparing the peak stress ratios, it became clear that the peak stress ratio of coral sand is about twice the peak stress ratio of Toyoura sand. In addition, when comparing the axial strains up to the peak stress ratio, the axial strain is about 5.0 to $6.0 \%$ for coral sand $\mathrm{A}$ and 4.5 to $6.0 \%$ for coral sand $\mathrm{B}$, the axial strain is 1.7 to $3.5 \%$ for Toyoura sand, It was found that about twice the axial strain is required to reach the peak stress ratio.

PIV analysis was performed from images taken during these experiments. Figure 7 shows the picture and the incremental contour of maximum shear strain when the principal stress ratio of Toyoura sand is peaked. The analysis results shown in the figure show the increase in maximum shear strain compared to the image with axial strain $0.1 \%$. Analysis was also carried out for coral sand and the slip angle of the shear band was obtained from these contour. In addition, shear resistance angle was obtained from Coulomb's failure criterion as cohesion is $0 \mathrm{kN} / \mathrm{m}^{2}$. The relationship between the shear band slip angle and the shear resistance angle is shown in Fig. 8. In the order of Toyoura sand, coral sand A and coral sand B, the shear band slip angles were $45.1^{\circ}, 50.6^{\circ}, 56.2^{\circ}$, respectively and the shear resistance angles were $64.5^{\circ}$, $58.9^{\circ}$ and $62.5^{\circ}$, respectively. From the graph of shear slip angle and shear resistance angle, it was clear that the relationship of $\alpha=\phi / 2+45^{\circ}$ does not hold clearly because $\alpha$ of the two types of coral sand is low. Figure 9 shows the result of examining the shear band slip angle $\alpha$ from the obtained shear resistance angle $\phi$ using Arthur, which are representative shear band slip angle calculation formulas. The horizontal axis shows the calculated value, and the vertical axis shows the experimental result. As it can be seen from the figure, the shear band slip angle of coral sand calculated by the Arthur's expression, it became clear that they agree roughly. From this, it was cleared that a new bearing capacity formula is needed to estimate shear slip angle $\alpha$ from shear resistance angle $\phi$, considering crushability and compressibility of coral sand. Finally, from the maximum shear strain increment contour obtained by PIV analysis, the relationship between the average value of the shear band width from the upper edge to the lower edge of the calculated image, its standard deviation and axial strain are shown in

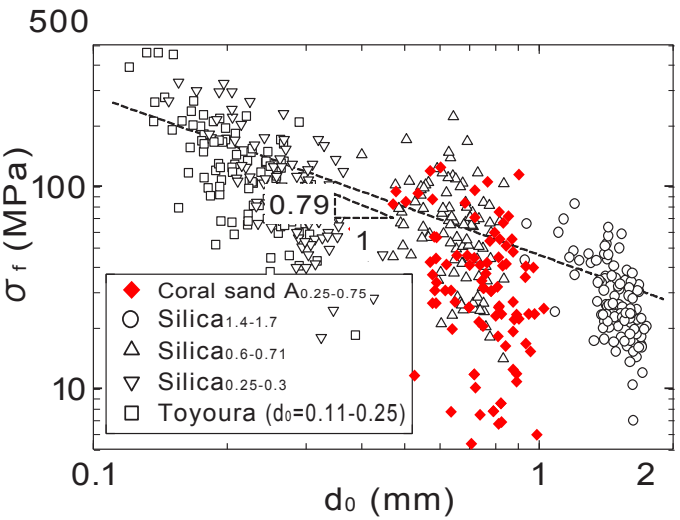

Fig. 5. Effect of particle size on single crushing strength (Retouched to Nakata et al., 2011 )

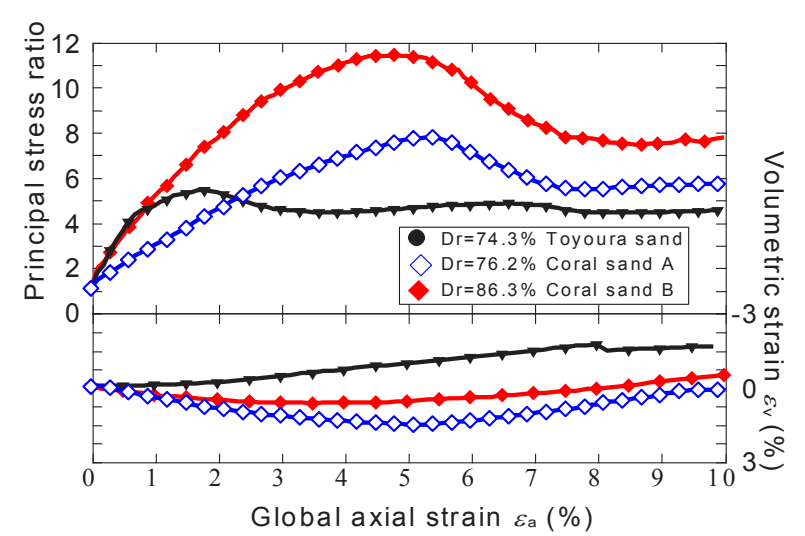

Fig. 6. Principal stress ratio - strain relationship

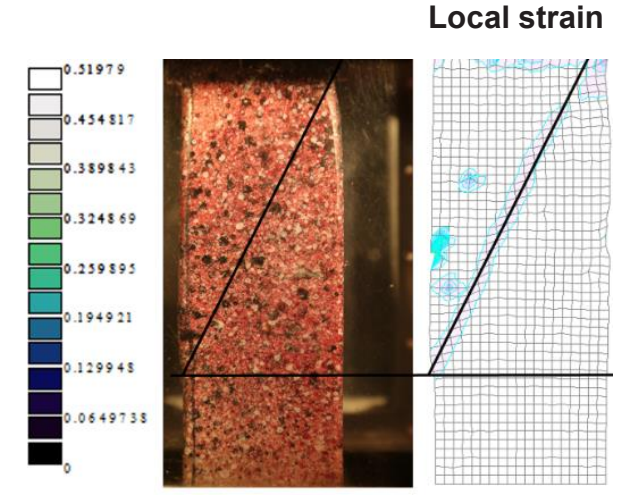

Fig. 7. Determination of shear band slip angle by contour

Figures 10 and 11. As shown in Figures 10 and 11, the average value of the shear band width at the peak of Toyoura sand was 10 to 20 times ( 2.20 to $2.40 \mathrm{~mm}$ ) of the average particle size, and the standard deviation was 0.1 to $0.9 \mathrm{~mm}$. The coral sand A was 7 to 10 times (3.36 to $4.80 \mathrm{~mm}$ ), the standard deviation was 0.9 to $1.6 \mathrm{~mm}$, the coral sand B was 5 to 8 times ( 5.5 to $8.8 \mathrm{~mm}$ ), the standard deviation was 1.4 to $1.9 \mathrm{~mm}$. From the figures, in the case of Toyoura sand, the width of the shear band is approximately in the range of 10 to 20 times the average particle diameter even after reaching the peak principal stress ratio, and the sample standard deviation decreases similarly with increasing the axial strain, it was found that shear bands are generated uniformly as the axial strain increases after the peak principal stress ratio is exerted. On the other hand, in the case of coral sand, which is a crushable material, the average value of the shear band 


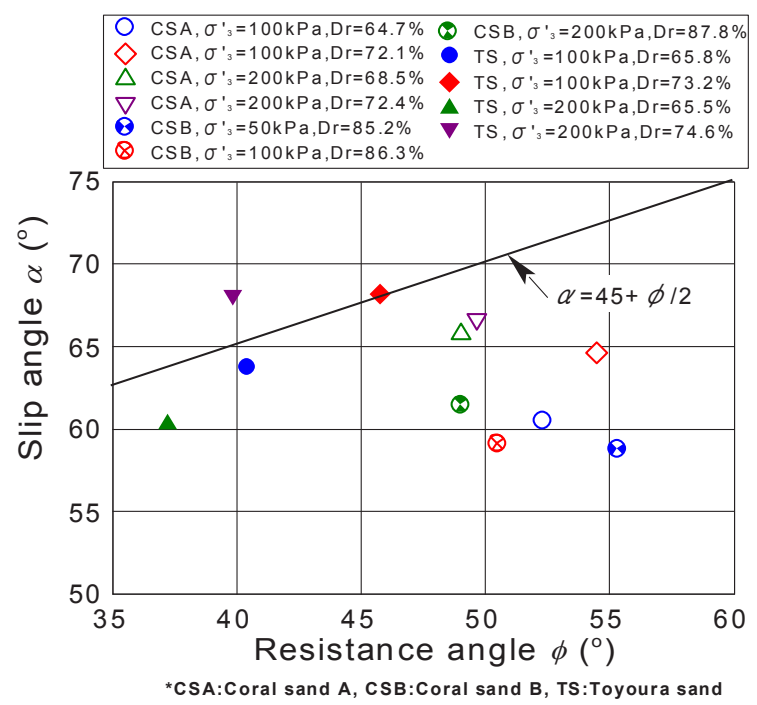

Fig. 8. Slip angle - resistance angle relationship

width is generally small in the coral sand A, and the sample standard deviation is slightly increasing. In the coral sand B, after reaching the peak principal stress ratio, the average value of the width of the shear band showed a tendency to rise, but no large difference was observed in the sample standard deviation. In the macroscopic behavior observed from Figure 6, in the case of Toyoura sand, the axial strain is shifted to the residual state at about $0.5 \%$ after reaching the peak principal stress ratio, whereas the coral sand, the axial strain is shifted to the residual state at about $2.0 \%$ after reaching the peak principal stress ratio. From these analysis results and Figure 6, comparing non-crushable materials and crushable materials, non-crushable materials such as Toyoura sand are rapidly destroyed and the maximum shear strain locally occurs near the shear band. On the contrary, the failure progresses gradually with crushable materials, and it is inferred that the shear band irregularly expands.

\section{Conclusion}

In this study, a series of experimental tests were conducted on three types of sand with different crushing characteristics for the purpose of grasping the shear band of sand under plane strain compression. The findings are summarized below.

(1) It was revealed that the crushing strength of coral sand B is about $1 / 3$ of that of quartz sand of the same grain size. (2) From the graph of shear slip angle $\alpha$ and shear resistance angle $\phi$, it became clear that $\alpha$ with using the Coulomb's equation $\alpha=\phi / 2+45^{\circ}$ is low for two kinds of coral sands, and it can be roughly calculated by using Arthur's equation.

(3) The ratio of the average value of the shear band width to the average grain size at the peak principal stress ratio was 10 to 20 times, 7 to 10 times, 5 to 8 times in the order of Toyoura sand, coral sand A, and coral sand B. The standard deviation was 0.1 to $0.9 \mathrm{~mm}, 0.9$ to $1.6 \mathrm{~mm}$, and 1.4 to $1.9 \mathrm{~mm}$.

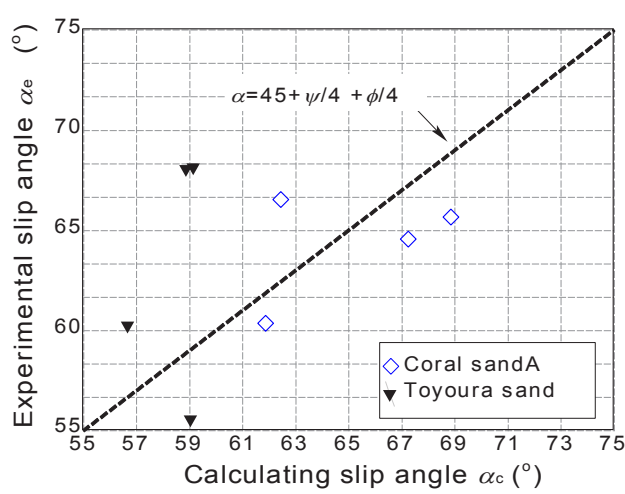

Fig. 9. Examining the shear band slip angle $\alpha$ from the obtained shear resistance angle $\phi$

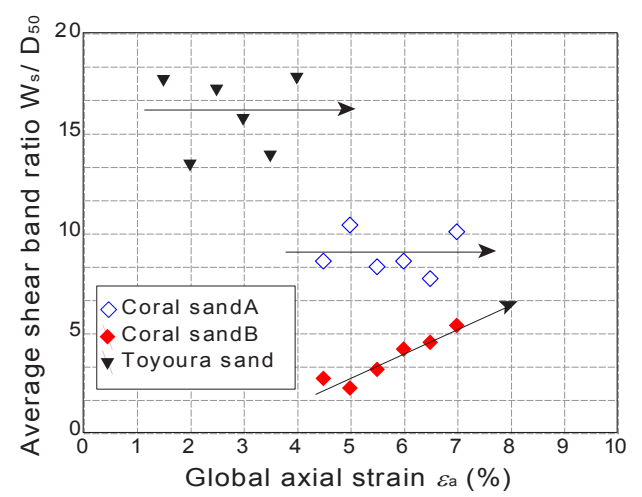

Fig. 10. Average shear band width ratio Average/ $\mathrm{D}_{50}-$ axial strain relationship

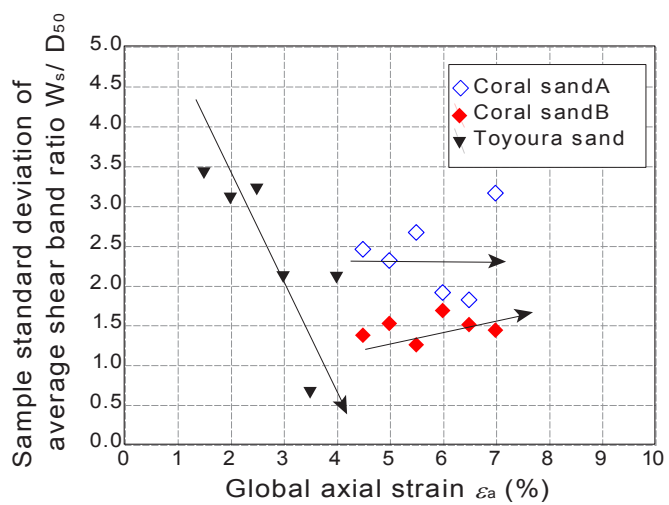

Fig. 11. Sample standard deviation of shear band width ratio Average/ $\mathrm{D}_{50}$ - axial strain relationship

\section{References}

1. K. Yagi and S. Miura, Jounal of JSCE, 757, 221-234 (2004) (in Japanese)

2. Y. Nakata, Y. Kato, M. Hyodo, A. F. L. Hyde, H. Murata, Soils and Foundations, Soils and Foundations, 4, 2, 39-51 (2001)

3. Y. Nakata et al., ibid.

4. J.R.F. Arthur, T. Dunstan, Q.A.J.L. Al-Ani, A. Assadi, Géotechnique, 27, 1, 53-74, (1977) 